\title{
Prácticas innovadoras: Uso de la plataforma blackboard en modalidades semipresenciales. Caso práctico UABC FIN Tecate.
}

Innovative practices: use of blackboard platform in blended modalities. END Case UABC Tecate

\author{
Velia Verónica Ferreiro Martínez \\ Universidad Autónoma de Baja California \\ vferreiro@uabc.edu.mx \\ Adriana Isabel Garambullo \\ Universidad Autónoma de Baja California \\ adriana.garambullo@uabc.edu.mx \\ Janette Brito Laredo \\ Universidad Autónoma de Baja California \\ jbrito@uabc.edu.mx
}

\section{Resumen}

La gran transformación profesional del siglo actual, es un gran desafío para Instituciones de Educación Superior que buscan responder a las necesidades del entorno adoptando para ello una didáctica diferente, centrada en la innovación educativa, el aprendizaje colaborativo y la investigación, basados en la educación a distancia con apoyo en las tecnologías virtuales de información y comunicación.

Este artículo, trata el tema de la realización de mejores prácticas dentro del proceso enseñanza aprendizaje al relatar la experiencia del uso de la plataforma virtual blackboard en el entorno de cursos semipresenciales ofertados por la Facultad de Ingeniería y Negocios Tecate de la Universidad Autónoma de Baja California, implementando las Tecnologías de Información y la Comunicación (TICs) aplicadas con el objetivo de involucrar a los estudiantes universitarios en tendencias educativas que 
les permitan adquirir conocimientos y capacitación dentro de su formación profesional y a la vez familiarizarse en el uso de la plataforma y sus herramientas. Esta experiencia se realizó dentro de un periodo semestral en grupos de quinto y sexto semestres de la etapa disciplinaria del Programa Educativo de Ingeniería Industrial.

Palabras clave: Innovación Educativa, Aprendizaje Colaborativo, TIC's, Mejores Prácticas, Tendencias Educativas, Educación a Distancia y Semipresencia

\section{Abstract}

The great professional transformation of this century, is a great challenge for higher education institutions seeking to respond to the needs of the environment thereby adopting different didactics focusing on educational innovation, research andthe collaborative learning, distance education basadosen with support virtual technologies of information and communication. This article addresses the issue of the implementation of best practices within the teaching-learning process to relate the experience of using the blackboard virtual platform environment learning courses offered by the Faculty of Engineering and Business Tecate Autonomous University of Baja California, implementing the Information Technology and Communication (ICT) applied with the aim of engaging college students in educational trends that allow them to acquire knowledge and training within their training and yet familiar in the use of the platform and its tools . This experiment was conducted within a six-month period in groups of fifth and sixth semesters three step disciplinary Industrial Engineering Education Program.

Key words: Educational Innovation Collaborative Learning, ICT, Best Practices, Educational Trends, Distance Education and Semipresencia

Fecha recepción: Enero 2013

Fecha aceptación: Abril 2013

\section{Introducción}


La gran transformación profesional del siglo actual, es un gran desafío para Instituciones de Educación Superior que buscan responder a las necesidades del entorno adoptando para ello una didáctica diferente, centrada en la innovación educativa, el aprendizaje colaborativo y la investigación, basados en la educación a distancia con apoyo en las tecnologías virtuales de información y comunicación. En este sentido es importante analizar la siguiente pregunta de investigación:

¿Cuál es la efectividad de utilización de la Plataforma Blackboard en la impartición de unidades de aprendizaje para estudiantes que cursan el Quinto y Sexto Semestre del Programa Educativo de Ingeniería Industrial perteneciente a la Facultad de Ingeniería y Negocios Tecate?

Bajo esta perspectiva el objetivo general de esta investigación consiste en conocer los beneficios que aporta la utilización de la Plataforma Blackboard en la impartición de unidades de aprendizaje en Instituciones de Educación Superior.

De lo anterior se desprenden a continuación los objetivos específicos:

1. Identificar la efectividad del uso de la Plataforma Virtual Blackboard para la Comunicación e Información.

2. Identificar los beneficios que aporta la utilización de la Plataforma Blackboard para contribuir a la Administración del Tiempo.

3. Identificar la efectividad de la Optimización de Recursos al utilizar la Plataforma Blackboard en unidades de aprendizaje.

La presente investigación pretende aportar información relevante al compartir las experiencias y resultados del uso de la plataforma blackboard dentro del proceso de 
enseñanza- aprendizaje, de tal manera que contribuya como una opción viable para una mejor administración de recursos en educación superior. La investigación se justifica ya que tradicionalmente, la educación se ha basado en modelos de tipo expositivo, en los cuales un experto en determinado tema transmite su conocimiento a otras personas por medio de contenidos teóricos. Las instituciones de educación superior necesitan entrar a la competitividad de hoy en día y esto se logra mediante la aplicación de mejores prácticas como la utilización de plataformas virtuales que contribuyan al logro de un aprendizaje significativo. Es por ello la importancia del estudio de la utilización de la plataforma blackboard como herramienta dentro del proceso enseñanza aprendizaje para que el docente y los estudiantes logren una mejor administración de su tiempo y optimización de recursos.

El presente estudio aportara evidencias para validar las siguientes hipótesis:

Hipótesis 1: La utilización de la Plataforma Blackboard facilita la Comunicación y transmisión de Información en la impartición de unidades de aprendizaje de Instituciones de Educación Superior.

Hipótesis 2: La utilización de la Plataforma Blackboard propicia la Administración del Tiempo en unidades de aprendizaje impartidas en educación superior.

Hipótesis 3: La utilización de la Plataforma Blackboard contribuye a la Optimización de Recursos en instituciones de Educación Superior.

Contenido:

CAPÍTULO I EDUCACIÓN A DISTANCIA, TECNOLOGÍAS VIRTUALES Y PLATAFORMA BLACKBOARD

Educación a distancia y cursos semipresenciales

La educación a distancia incluye aquellos programas en ambientes educativos que implican la separación espacio temporal de profesores y alumnos, donde se 
desarrollan y articulan elementos del proceso enseñanza aprendizaje mediante el uso de materiales didácticos y tecnologías de la información y la comunicación (TICs) para propiciar iteraciones sincrónicas y asincrónicas (Ferrando, Moreno, 2004).

La educación a distancia se caracteriza por la flexibilidad de sus horarios, pues el mismo estudiante organiza su tiempo de estudio, lo cual requiere cierto grado de autodisciplina. Esta flexibilidad de horarios a veces está limitada en ciertos cursos que exigen participación en línea en horarios o espacios específicos. Otra característica de la educación a distancia es el uso de las Tecnologías de la Información y la Comunicación (TIC) para formar comunidades o redes de estudio donde los individuos pueden interactuar, para discutir sobre diversos temas y a la vez adquirir conocimientos y modernas herramientas de trabajo. También es imprescindible tener una nueva visión de los roles que desempeñan los maestros y los estudiantes en esta modalidad de estudio, el maestro deja de ser el protagonista, convirtiéndose en un facilitador del proceso educativo y le cede el paso al estudiante, el cual debe tener un compromiso firme con su propio proceso de formación.

Existen una diversidad de opiniones de autores en cuanto a las ventajas y desventajas de la educación a distancia, entre las cuales podemos resumir las presentadas en la siguiente tabla:

Tabla 1 Ventajas y desventajas de la educación a distancia

\begin{tabular}{|l|l|}
\hline \multicolumn{2}{|l|}{ Educación a distancia } \\
\hline Ventajas & Desventajas \\
\hline Elimina las barreras geográficas, la población & Dificulta transmitir y conservar determinados \\
puede acceder a este tipo de educación & contenidos actitudinales para mejorar la \\
independientemente de donde resida. & socialización. \\
\hline Proporciona flexibilidad en el horario. & Generalmente el cambio a un sistema de \\
\hline
\end{tabular}




\begin{tabular}{|c|c|}
\hline & $\begin{array}{l}\text { educación a distancia exige al alumnado una } \\
\text { adaptación específica: ha de aprender a usar } \\
\text { materiales didácticos específicos y aulas } \\
\text { virtuales, a comunicarse con sus profesores y } \\
\text { con otros alumnos a través de medios de } \\
\text { comunicación y ha de ser capaz de organizar } \\
\text { su tiempo de estudio para compaginar vida } \\
\text { personal, laboral y académica. }\end{array}$ \\
\hline $\begin{array}{l}\text { Reduce costos al evitar gastos de traslados o } \\
\text { residencia en un lugar diferente. }\end{array}$ & $\begin{array}{l}\text { Al eliminarse la interacción social en } \\
\text { presencia la comunicación se reduce a un solo } \\
\text { canal y resulta menos profunda, por lo que es } \\
\text { posible que el alumno se aislé y desmotive, } \\
\text { ante ello, es necesaria una intervención activa } \\
\text { del profesor tutor. }\end{array}$ \\
\hline $\begin{array}{l}\text { Incorpora herramientas tecnológicas para el } \\
\text { manejo de la información. }\end{array}$ & $\begin{array}{l}\text { Posibles retrasos en la retroalimentación } \\
\text { (feedback) y rectificación de posibles errores. }\end{array}$ \\
\hline $\begin{array}{l}\text { El alumno desarrolla una alta capacidad para } \\
\text { autorregular su propio aprendizaje } \\
\text { favoreciendo así sus actitudes y valores de } \\
\text { responsabilidad, disciplina y compromiso para } \\
\text { lograr ser autónomo. }\end{array}$ & \\
\hline
\end{tabular}

Fuente: Educación a distancia. Recuperado el 04 de diciembre del 2012, de http://es.wikipedia.org/wiki/Educaci\%C3\%B3n_a_distancia.

Educación semipresencial

La educación semipresencial es una nueva herramienta cuya metodología consiste en impartir clases en línea complementadas con lecciones presenciales. Las clases se realiza en una plataforma web, desde la cual se pueden visualizar los contenidos, descargar las clases, presentaciones, podcasts, materiales del curso, etcétera (Pardo, 2003). En esta modalidad, los estudiantes puedan acceder a una educación sin la necesidad de presentarse físicamente en la institución educativa todos los días. Por lo tanto, es una excelente opción para quienes trabajan, o bien, para aquellos que su labor en casa no les permite estar mucho tiempo en la escuela. Esto 
con la ventaja de tener una educación sin las limitaciones de un horario o lugar, ya que en cualquier parte la persona puede estudiar con la única condición de que tiene que asistir periódicamente a tutoría para aclarar dudas, realizar actividades de evaluación o tomar alguna clase o materia. La ventaja que tiene este modelo es que el alumno va desarrollando su aprendizaje de una manera un poco más autónoma, ya que la investigación es indispensable. En otras palabras, el estudiante es capaz de regir y proyectar el proceso de su propio aprendizaje.

En la educación semipresencial el estudiante asiste periódicamente a sesiones en la universidad o instituto, con el fin de estar al corriente de las actividades de los alumnos, atender sus dudas e inquietudes, aumentar la formalidad, poner en práctica ciertos conocimientos, entre otros. Estas horas, por ser pocas, se limitan a orientaciones por parte de los profesores y a resolver dudas. La eficacia del programa se debe a la eficiencia del alumno, es decir, se determina según la capacidad del alumno para organizar su propio tiempo y ritmo de estudio. El aprendizaje se monitorea por medio de tutorías, las cuales pueden ser de dos tipos: presenciales y virtuales. La evaluación se implementa de acuerdo a cada curso. Existen clases virtuales que pueden ser de una a más veces por semana; y presenciales, que se destinan regularmente para realizar exámenes parciales y finales. Las ventajas y desventajas de la educación semipresencial se enlistan en la tabla 2.

Tabla 2 Ventajas y desventajas de la educación semipresencial

\begin{tabular}{|l|l|}
\hline \multicolumn{2}{|l|}{ Educación semipresencial } \\
\hline Ventajas & Desventajas \\
\hline Mayor Flexibilidad e interactividad entre & $\begin{array}{l}\text { Se podría generar una desconfianza ante } \\
\text { la falta de comunicación concurrida entre } \\
\text { los alumnos y las tecnologías. }\end{array}$ \\
& $\begin{array}{l}\text { el profesor y el alumno, sobre todo en el } \\
\text { proceso de evaluación de aprendizaje del } \\
\text { alumno }\end{array}$ \\
\hline
\end{tabular}




\begin{tabular}{|c|c|}
\hline $\begin{array}{l}\text { Mejor y mayor acceso a grandes } \\
\text { cantidades de información }\end{array}$ & $\begin{array}{l}\text { Aislamiento que se puede llegar a dar } \\
\text { entre seres humanos, eliminando la } \\
\text { interacción social física. Puede influir para } \\
\text { bien o para mal (dependiendo del caso de } \\
\text { cada persona en su forma de ser y pensar) } \\
\text { al querer interactuar y desarrollarse en el } \\
\text { ámbito laboral y social dependiendo su } \\
\text { carrera, todo esto al no haber obtenido } \\
\text { suficiente contacto social. }\end{array}$ \\
\hline $\begin{array}{l}\text { Individualización del aprendizaje al ritmo } \\
\text { de cada alumno y ahorro de dinero, el } \\
\text { alumno no tiene que desplazarse a la } \\
\text { institución educativa }\end{array}$ & $\begin{array}{l}\text { La pasividad del alumno frente a este } \\
\text { medio, pues lo puede percibir como un } \\
\text { "medio fácil". }\end{array}$ \\
\hline
\end{tabular}

Fuente: Educación a distancia. Recuperado el 04 de diciembre del 2012, de http://es.wikipedia.org/wiki/Educaci\%C3\%B3n_a_distancia

Tecnologías virtuales de información y comunicación (TICs)

Se denominan Tecnologías de la Información y las Comunicación al conjunto de tecnologías que permiten la adquisición, producción, almacenamiento, tratamiento, comunicación, registro y presentación de informaciones, en forma de voz, imágenes y datos contenidos en señales de naturaleza acústica, óptica o electromagnética. Las TICs incluyen la electrónica como tecnología base que soporta el desarrollo de las telecomunicaciones, la informática y el audiovisual (Rosario, Jimmy, 2005).

El mismo autor menciona las principales características de las TICs:

Inmaterialidad:(Posibilidad de digitalización). Las TICs convierten la información, tradicionalmente sujeta a un medio físico, en inmaterial. Mediante la digitalización es posible almacenar grandes cantidades de información, en dispositivos físicos de 
pequeño tamaño (discos, CD, memorias USB, etc.). A su vez los usuarios pueden acceder a información ubicada en dispositivos electrónicos lejanos, que se transmite utilizando las redes de comunicación, de una forma transparente e inmaterial. Esta característica, ha venido a definir lo que se ha denominado como "realidad virtual", esto es, realidad no real. Mediante el uso de las TICs se están creando grupos de personas que interactúan según sus propios intereses, conformando comunidades o grupos virtuales.

Instantaneidad: Podemos transmitir la información instantáneamente a lugares muy alejados físicamente, mediante las denominadas "autopistas de la información".

Se han acuñado términos como ciberespacio, para definir el espacio virtual, no real, en el que se sitúa la información, al no asumir las características físicas del objeto utilizado para su almacenamiento, adquiriendo ese grado de inmediatez e inmaterialidad.

Aplicaciones Multimedia: Las aplicaciones o programas multimedia han sido desarrollados como una interfaz amigable y sencilla de comunicación, para facilitar el acceso a las TICS de todos los usuarios. Una de las características más importantes de estos entornos es "La interactividad". Es posiblemente la característica más significativa. A diferencia de las tecnologías más clásicas (TV, radio) que permiten una interacción unidireccional, de un emisor a una masa de espectadores pasivos, el uso del ordenador interconectado mediante las redes digitales de comunicación, proporciona una comunicación bidireccional (sincrónica y asincrónica), personapersona y persona- grupo.

Otra de las características más relevantes de las aplicaciones multimedia, y que mayor incidencia tienen sobre el sistema educativo, es la posibilidad de transmitir información a partir de diferentes medios (texto, imagen, sonido, animaciones, etc.). Por primera vez, en un mismo documento se pueden transmitir informaciones multisensoriales, desde un modelo interactivo. 


\section{Plataforma blackboard}

Una plataforma educativa es una herramienta ya sea física, virtual o una combinación físico-virtual, que brinda la capacidad de interactuar con uno o varios usuarios con fines pedagógicos. Además, se considera un proceso que contribuye a la evolución de los procesos de aprendizaje y enseñanza, que complementa o presenta alternativas en los procesos de la educación tradicional (Rodríguez. Diéguez. Saénz Barrio).

En la actualidad, la mayor parte de las plataformas educativas son programas computacionales (software) o equipos electrónicos (hardware). Blackboard Learning System $^{\mathrm{Tm}}$ es una plataforma que se utiliza para los cursos semipresenciales o a distancia. Es una plataforma computacional, flexible, sencilla e intuitiva que se utiliza en muchas universidades de los Estados Unidos de América y contiene las funciones básicas para crear los contenidos y documentos que se necesitan para la administración de un curso, usa Internet como medio.

Esta plataforma ayuda a mejorar todos los aspectos de la experiencia educativa. La plataforma permite: Mejorar el desempeño individual e institucional, hacer la enseñanza y el aprendizaje más eficaz en el aula y más allá, tomar decisiones más informadas y mejorar los resultados, ofrecer una más atractiva, la experiencia interactiva de aprendizaje individualizado, hacer la vida dentro y fuera de la escuela más conveniente y segura, prestar servicios y experiencias que satisfagan las nuevas expectativas de los alumnos.

Desde sus comienzos, el Blackboard Learning System ${ }^{\text {тм }}$ fue diseñado para instituciones dedicadas a la enseñanza y el aprendizaje. La tecnología y los recursos Blackboard $^{\circledR}$ son el motor de los programas educativos híbridos, optimizados por Internet, en más de 2.000 instituciones académicas. Se trate de una universidad de investigación, college comunitario, escuela secundaria o programa virtual de MBA, el Blackboard Learning System ofrece una solución comprobada para satisfacer las 
necesidades de la institución. El Blackboard Learning System [1] presenta un ambiente premiado para la enseñanza y el aprendizaje en Internet que proporciona la infraestructura para la optimización y soporte de:

Administración de cursos: El Blackboard Learning System elimina el estrés de la gestión de administración de cursos, ya sea a través de recursos que pueden ser usados por instructores, administradores individuales, o a través de procesos integrados con otros sistemas en la infraestructura de TI.

Herramientas instructivas: El Blackboard Learning System ofrece una amplia variedad de características para facilitar la creación de contenido profesional y de alta calidad, junto con herramientas complementarias que optimizan aún más el plan de estudios general del curso.

Colaboración y Comunicación: El Blackboard Learning System es un ambiente completo para la colaboración en línea, con características que incluyen:

- Conversaciones libres, disertaciones a través de chat, chats de preguntas y respuestas, archivos, espacios para debate, whiteboarding, tours de la clase y navegación grupal en Internet para una colaboración sincronizada basada en la Web, incluyendo grabado y almacenaje de sesiones.

- Calendario y Agenda incorporados, así como funcionalidad de Avisos.

- Blackboard Messages facilita la comunicación entre usuarios de Blackboard sin necesidad de una cuenta externa de correo electrónico.

Estimación y Evaluación: El Blackboard Learning System ofrece la autoría dinámica de estimación basada en reglas con menos clics y más funcionalidad. 
Cada una es la mejor solución de su clase y son más poderosas juntas a través de una arquitectura compartida, interfaces uniformes, su capacidad de compartir archivos con precisión y características de administración robusta. Blackboard Academic Suite proporciona una experiencia educativa integrada para alumnos, profesores y personal, y una visión de gestión integrada.

\section{CAPÍTULO III METODOLOGÍA}

La presente investigación es de tipo cuantitativa ya que utiliza información a través de una encuesta basada en la escala de Likert, cuyo objeto es recoger, procesar y analizar características que se dan en un grupo determinado pretendiendo medir de manera objetiva y con la mayor precisión posible las variables involucradas. El diseño es descriptiva correlacional de acuerdo a la forma en que se analizaron los datos, ya que el propósito es decir cómo es y cómo se manifiesta determinado fenómeno (Hernández Sampieri, Fernández Collado y Baptista Lucio, 2006). Además, es un estudio de campo o investigación directa ya que se efectúa en el lugar y tiempo en que ocurre el fenómeno objeto de estudio (Zorrilla Arena, 2007).

La población objeto de estudio a la cual se refiere la investigación estuvo compuesta por 24 estudiantes matriculados en quinto y sexto semestres del Programa Educativo de Ingeniería Industrial de la Facultad de Ingeniería y Negocios Tecate, en la Universidad Autónoma de Baja California, en el periodo 2012-2 (Agosto-Diciembre). La población representa el cien por ciento de la población seleccionada.

Retomando el problema de investigación ¿Cuál es la efectividad de utilización de la Plataforma Blackboard en la impartición de unidades de aprendizaje para estudiantes que cursan el Quinto Sexto Semestre del Programa Educativo de Ingeniería Industrial perteneciente a la Facultad de Ingeniería y Negocios Tecate? se 
plantean a continuación las hipótesis nulas guiadoras de la presentación de esta sección.

Ho1: La utilización de la Plataforma Blackboard no facilita la Comunicación y transmisión de Información en la impartición de unidades de aprendizaje de Instituciones de Educación Superior.

Ho2: La utilización de la Plataforma Blackboard no propicia la Administración del Tiempo en unidades de aprendizaje impartidas en educación superior.

Ho3: La utilización de la Plataforma Blackboard no contribuye a la Optimización de Recursos en instituciones de Educación Superior.

Variables

Para determinar qué es lo que se requiere medir, cómo se define, cómo se observará y la forma de medirlo, se construyó una matriz de congruencia en la cual se incluyó la pregunta de investigación, objetivo general y objetivos específicos e hipótesis para determinar las variables en estudio. A continuación se presentan las definiciones conceptuales de las variables en estudio:

\section{Comunicación e Información}

De acuerdo a la Real Academia Española la información está constituida por un grupo de datos ya supervisados y ordenados, que sirven para construir un mensaje basado en un cierto fenómeno o ente. La información permite resolver problemas y tomar decisiones, ya que su aprovechamiento racional es la base del conocimiento.

Según Córicala comunicaciones un proceso en el que se produce un intercambio modificador para los involucrados, y que tiene como elementos un emisor, un receptor, un canal, un código y algunos elementos que pueden aparecer, como el 
medio y el ruido. Sin entrar en mayores detalles sobre el proceso de comunicación en sí, digamos que en la comunicación humana, emisores y receptores intercambian sus roles alternativamente, produciéndose lo que en la jerga comunicacional se denomina feedback o realimentación de contenido y consiste en que la respuesta del primer receptor se constituye en agente modificador de la nueva emisión del emisor original.

Administración del Tiempo

La administración del tiempo es revisar y analizar nuestros conceptos sobre la correcta aplicación del tiempo (Stack 2012). Administrar el tiempo significa administrar las actividades de uno mismo. Esto implica saber tomar decisiones e implementarlas. Hacer las cosas oportunamente. Saber que uno puede controlar el tiempo y la calidad de su propia vida. Examinar nuestras actitudes y proponernos un cambio. Para llegar a ser eficiente, debo tener una actitud optimista. Ser disciplinado y desear serlo. Aceptar que no hay tiempo para hacerlo todo, pero que siempre existe tiempo, para hacer las cosas más importantes.

Optimización de Recursos

De acuerdo a Asturias, 2008 optimizar, no es sinónimo de ahorrar o suprimir, se define en términos generales como buscar la mejor manera de realizar una actividad. En el mundo empresarial la optimización de recursos tienen que ver con la eficiencia (utilizar los recursos de la mejor manera posible, o dicho de otra forma, obtener los mayores beneficios con los mínimos costes). Pero la eficiencia tiene estrecha conexión con la eficacia (término que hace énfasis en los resultados, hacer las cosas correctas, lograr objetivos, crear más valores), por lo tanto para optimizar recursos, no nos bastaría con ser eficientes, sino que también hay que ser eficaces.

El instrumento utilizado para medir las variables de estudio fue un cuestionario construido ex profeso, el cual contiene en la primera sección datos característicos con el objetivo de obtener información específica del grupo de estudio. La parte principal 
del instrumento consta de 15 declaraciones agrupadas en tres factores teóricos, ante los cuales se solicita la reacción de los individuos respecto a las variables a medir usando la escala Likert, de cinco alternativas de opinión para valorarlas: totalmente en desacuerdo, en desacuerdo, ni en acuerdo ni desacuerdo, de acuerdo y totalmente de acuerdo.

El instrumento preparado para la recolección de los datos fue aplicado a los estudiantes de quinto semestre matriculados en los cursos de Ingeniería de Métodos e Investigación de Operaciones, así como a los de sexto de Administración de Calidad y Estudio del Trabajo; lo cual permitió conocer las opiniones que tienen sobre la utilización de la plataforma blackboard en cursos semipresenciales.

Para realizar un análisis de los resultados se elaboró una tabla que muestra la media aritmética de cada una de las declaraciones del instrumento de observación, con el propósito de obtener mayor información descriptiva acerca de la opinión de los alumnos. Y de esta manera determinar si se cumple las hipótesis de investigación, considerando una escala de 0 a 5 para medir cada variable.

\section{CAPÍTULO IV ANÁLISIS DE LOS RESULTADOS}

En esta sección se presentan los resultados obtenidos al analizar las respuestas de la encuesta aplicada a los estudiantes de quinto y sexto semestre del programa educativo de Ingeniería Industrial referente a la información característica de la población objeto de estudio (ver Tabla 3) y a los aspectos relacionados con cada una de las variables de investigación, así como las principales recomendaciones determinadas según dichos resultados.

Tabla 3 Información característica de la población objeto de estudio 


\begin{tabular}{|c|c|c|c|c|}
\hline $\begin{array}{l}\text { Datos } \\
\text { Personales: }\end{array}$ & & & & \\
\hline Edad & $\begin{array}{l}\text { 18-20 años: } 34 \\
\%\end{array}$ & $\begin{array}{l}\text { 21-23 años: } \\
58 \%\end{array}$ & 24-25 años: 8\% & $\begin{array}{l}\text { Más de } 25 \\
\text { años: } 0 \%\end{array}$ \\
\hline Género & $\begin{array}{l}\text { Masculino: } \\
54 \%\end{array}$ & $\begin{array}{l}\text { Femenino: } \\
46 \%\end{array}$ & & \\
\hline Trabaja & $\begin{array}{ll}67 \% & \text { No } \\
\text { Trabaja } & \end{array}$ & 33\% Si Trabaja & $\begin{array}{l}\text { 88\% Trabaja en } \\
\text { Empresa }\end{array}$ & $12 \%$ en Otro \\
\hline $\begin{array}{l}\text { Semestre y } \\
\text { materias que } \\
\text { cursa }\end{array}$ & 54\% Quinto & $46 \%$ Sexto & $\begin{array}{l}\text { 54\% Ingeniería } \\
\text { de Métodos y } \\
50 \% \\
\text { Investigación } \\
\text { de Operaciones }\end{array}$ & $\begin{array}{l}46 \% \\
\text { Administración } \\
\text { de } \quad \text { Calidad } \\
\text { como Estudio } \\
\text { del Trabajo }\end{array}$ \\
\hline $\begin{array}{l}\text { Promedio } \\
\text { general de la } \\
\text { carrera }\end{array}$ & $\begin{array}{l}8 \% \text { entre 90- } \\
100\end{array}$ & $\begin{array}{l}67 \% \text { entre } 80- \\
89\end{array}$ & $\begin{array}{l}25 \% \text { entre70- } \\
79\end{array}$ & $\begin{array}{l}0 \% \text { Menos de } \\
70\end{array}$ \\
\hline
\end{tabular}

Esta tabla presente los resultados obtenidos en las encuestas aplicadas en cuanto a información general de los estudiantes de quinto y sexto semestre de la carrera de Ingeniería Industrial de la Facultad de Ingeniería y Negocio, mostrando que el $58 \%$ de la población objeto de estudio tiene entre 21 y 23 años, el 54\% es de género masculino y por consecuencia el otro $46 \%$ femenino, además; el $33 \%$ de los estudiantes encuestados trabaja, siendo el $88 \%$ en empresas y el $67 \%$ llevan al momento un promedio de calificaciones en su carrera de entre 80 a 89.

En la Tabla 4 se observan los ítems que abarcan cada una de las variables de esta investigación: Información y comunicación, administración del tiempo y optimización de recursos, así como los resultados obtenidos según la percepción de la población de este estudio. 
Tabla 4 Media aritmética de cada una de las declaraciones.

\begin{tabular}{|c|c|c|c|}
\hline Declarac & ión & & \\
\hline & & $\begin{array}{l}\text { El trabajar con la Plataforma Blackboard } \\
\text { incrementó la interacción y comunicación } \\
\text { entre estudiantes y con el profesor. }\end{array}$ & 3.71 \\
\hline & & $\begin{array}{l}\text { La plataforma blackboard cuenta con } \\
\text { herramientas como foros de debate, chats, } \\
\text { diarios, anuncios, entre otros, que facilitan la } \\
\text { comunicación entre estudiantes y el profesor. }\end{array}$ & 4.63 \\
\hline & & $\begin{array}{l}\text { La plataforma blackboard me ayuda a estar } \\
\text { informado sobre el contenido general del curso } \\
\text { y los criterios de evaluación. }\end{array}$ & 4.50 \\
\hline & & $\begin{array}{l}\text { A través de la plataforma blackboard me } \\
\text { entero en tiempo y forma de las actividades a } \\
\text { realizar durante el curso. }\end{array}$ & 4.38 \\
\hline & $\begin{array}{l}\text { Información y } \\
\text { comunicación }\end{array}$ & $\begin{array}{l}\text { yonsidero que la comunicación en la } \\
\text { ylataforma Blackboard entre alumno y } \\
\text { profesor es adecuada. }\end{array}$ & 4.00 \\
\hline & & $\begin{array}{l}\text { La utilización de la plataforma blackboard me } \\
\text { permite administrar mi tiempo para la entrega } \\
\text { de trabajos académicos. }\end{array}$ & 4.00 \\
\hline & 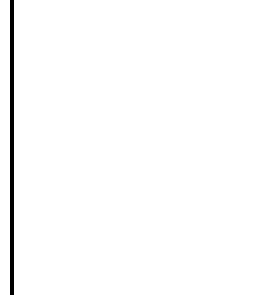 & $\begin{array}{l}\text { La Educación a través de la plataforma } \\
\text { blackboard implica amplias posibilidades de } \\
\text { participación de estudiantes dispersos con un } \\
\text { alto grado de autonomía de tiempo. }\end{array}$ & 3.79 \\
\hline Variables & $\begin{array}{l}\text { Administració } \\
\text { del tiempo }\end{array}$ & $\mid \begin{array}{l}\text { Los cursos semipresenciales me permiten } \\
\text { acomodarme a mis necesidades en función a la } \\
\text { carga de trabajo de la asignatura y mis } \\
\text { responsabilidades sociales y familiares. }\end{array}$ & 4.08 \\
\hline
\end{tabular}




\begin{tabular}{|c|c|c|}
\hline & $\begin{array}{l}\text { La distribución del tiempo asignado a los } \\
\text { trabajos en la plataforma Blackboard es } \\
\text { adecuado. }\end{array}$ & 4.17 \\
\hline & $\begin{array}{l}\text { Distribuí adecuadamente el tiempo para la } \\
\text { entrega de los trabajos en el curso } \\
\text { semipresencial a través de la plataforma } \\
\text { blackboard. }\end{array}$ & 3.67 \\
\hline & $\begin{array}{l}\text { Considero que la utilización de la plataforma } \\
\text { blackboard contribuye a optimizar los recursos } \\
\text { de mi facultad. }\end{array}$ & 4.29 \\
\hline & $\begin{array}{l}\text { La Educación a través de la plataforma } \\
\text { blackboard implica amplias posibilidades de } \\
\text { acceso y participación de estudiantes dispersos } \\
\text { aprovechando espacios virtuales. }\end{array}$ & 4.25 \\
\hline & $\begin{array}{l}\text { La utilización de la plataforma promueve el uso } \\
\text { de las TICS generando mecanismos de } \\
\text { interacción entre grupos de estudiantes y el } \\
\text { docente de manera semipresencial. }\end{array}$ & 3.88 \\
\hline & $\begin{array}{l}\text { La utilización de la plataforma permite el } \\
\text { ahorro en impresiones con el envió de } \\
\text { materiales educativos y trabajos de forma } \\
\text { digital. }\end{array}$ & 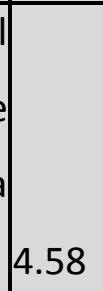 \\
\hline $\begin{array}{l}\text { Optimización } \\
\text { de recursos }\end{array}$ & $\begin{array}{l}\text { Considero que la plataforma Blackboard es una } \\
\text { herramienta útil para optimizar mis recursos } \\
\text { académicos al no requerir tanta inversión } \\
\text { económica en impresiones de todos los } \\
\text { trabajos, tareas, etcétera. }\end{array}$ & 4.79 \\
\hline
\end{tabular}

Fuente: Elaboración propia con base a los resultados obtenidos en cada uno de los ítems del cuestionario aplicado. 
Para observar el comportamiento de las variables, se presenta la media estadística resultante del constructo de preguntas que integra cada variable (Ver Tabla 5).

Tabla 5: Resultados por variables

\begin{tabular}{|l|l|}
\hline Variables & Resultado \\
\hline Información y comunicación & $=4.24$ \\
\hline Administración del tiempo & $=3.94$ \\
\hline Optimización de recursos & $=4.36$ \\
\hline
\end{tabular}

Fuente: Elaboración propia con base a los resultados obtenidos en cada uno de los ítems del cuestionario aplicado.

Para determinar el nivel de aceptación de cada hipótesis, se realizó un análisis estadístico, el cual consistió en determinar la media aritmética de cada variable, considerando una escala de 0 a 5, donde de acuerdo a la escala de Likert, consiste en cinco alternativas de opinión para valorarlas: totalmente en desacuerdo, en desacuerdo, indeciso, de acuerdo y totalmente de acuerdo. A continuación se enuncian nuevamente las hipótesis nulas del estudio y se presentan los resultados obtenidos para cada una de ellas.

Hipótesis nula 1

La hipótesis nula Ho1 declara que la utilización de la Plataforma Blackboard no facilita la Comunicación y transmisión de información en la impartición de unidades de aprendizaje de Instituciones de Educación Superior.

La media aritmética de la variable información y comunicación es de $=4.24$, lo que significa que los alumnos consideran que la utilización de la plataforma facilita la transmisión de información y una mejor comunicación por lo que se rechaza la hipótesis nula y se acepta la hipótesis de investigación en cuanto a esta variable. 
Hipótesis nula 2

La hipótesis nula Ho2 declara que la utilización de la Plataforma Blackboard no propicia la Administración del Tiempo en unidades de aprendizaje impartidas en educación superior.

La variable administración del tiempo $=3.94$ representa un factor importante que indica que con la utilización de la plataforma virtual los estudiantes y docentes pueden trabajar con la administración de su tiempo por lo que se rechaza parcialmente la hipótesis nula y se acepta la hipótesis de investigación.

Hipótesis nula 3

La hipótesis nula Ho3 declara que la utilización de la Plataforma Blackboard no contribuye a la Optimización de Recursos en instituciones de Educación Superior.

Como se puede observar la variable optimización de recursos refleja una media aritmética de $=4.36$, lo cual se considera elevado, ya que el intervalo es de 0 a 5 podemos concluir que se rechaza la hipótesis nula y se acepta la de investigación.

[1]http://www.blackboard.com/

\section{Conclusión}

\section{CAPITULO V CONCLUSIONES Y RECOMENDACIONES}

Según los resultados obtenidos en esta investigación podemos concluir que el uso de la plataforma blackboard es una herramienta importante y efectiva en la impartición de unidades de aprendizaje en Instituciones de Educación, ya que apoya la comunicación 
y el flujo de información entre estudiantes y el docente, además de contribuir a la administración del tiempo y la utilización óptima de recursos tanto por parte de los estudiantes, docentes y de la propia institución.

La principal recomendación en base a los resultados y conclusiones obtenidas, que se presenta a continuación pretende ser un factor para la mejora del proceso enseñanza aprendizaje en instituciones de educación superior.

La utilización de la plataforma blackboard ayuda en la mejora de la comunicación y transmisión de información, apoya tanto a los docentes como a los alumnos en la administración del tiempo y la optimización de los recursos, por lo tanto se recomienda implementar esta herramienta en la impartición de unidades de aprendizaje en instituciones de educación superior. Se espera que el esfuerzo realizado en esta investigación sea de beneficio para todos aquellos que estén interesados en implementar mejores prácticas como herramientas que permitan mejorar el aprendizaje.

\section{Bibliografía}

Ferrando Bravo, G.; Moreno Bonett, A. (2004). Educación continua a distancia: modelos, entornos, desarrollo y especificaciones. RIED. Revista Iberoamericana de Educación a Distancia, 7 (1), 133-146.

Hernández Sampieri, R., Fernández Collado, C. y Baptista Lucio, P. (2006). Metodología de la investigación. México: McGraw-Hill.

Lloréns, L., Figueroa, C., Espinosa, Y., Perezchica, E., Gaona, T., Sepúlveda, J. y Lizalde, F. (2009). Formación de redes de colaboración para la difusión de estrategias de educación abierta y a distancia apoyadas en tecnologías de información, comunicación y colaboración. Conferencia Conjunta Iberoamericana sobre Tecnologías para el Aprendizaje (pp. 223-232). Yucatán, México: Universidad Autónoma de Yucatán. 
Magíster José Luis Córica. (2012). COMUNICACIÓN Y NUEVAS TECNOLOGÍAS: SU INCIDENCIA EN LAS ORGANIZACIONES EDUCATIVAS, “Concepto de comunicación educativa"[Documento en PDF]. Recuperado de http://www.uaeh.edu.mx/docencia/VI_Lectura/maestria/documentos/LECT46.pdf.

Martínez, R., Heredia, Y. (2010). Tecnología educativa en el salón de clase estudio retrospectivo de su impacto en el desempeño académico de estudiantes universitarios del área de informática. Revista mexicana de investigación educativa 15 (45), 371-390.

Stack Laura (2012). What To Do When There's Too Much To Do. Berrett-Koehler Pub.

Vidal, M. P. (2004). Uso y Evaluación de la Plataforma de Enseñanza-Aprendizaje Virtual U. Revista de Medios y Educación, 24, 89-100.

Zorrilla, S. (2007). Introducción a la metodología de la investigación. México: Aguilar León y Cal edrs. 